\title{
MATHEMATICAL MODELING OF THERMAL MODES OF CLOSED TWO-PHASE THERMOSYPHONS REFRIGERANT R600A
}

\author{
A.S. Krasnoshlykov ${ }^{1,}$ a , Y.A. Zagromov ${ }^{1}$ \\ ${ }^{1}$ National Research Tomsk Polytechnic University, 634050 Tomsk, Russia
}

\begin{abstract}
Numerical analysis using the software package ANSYS FLUENT has been carried out. Characteristic temperature distribution, streamlines and velocity vectors for various heat loads have been obtained. The study found the possibility of using the software package for analysis of the energy transfer processes at high thermal loads.
\end{abstract}

\section{Introduction}

Thermosyphons and heat pipes are promising tools cooling and thermal control for various high energy-efficient equipment $[1,2]$. However, thermosyphons (TS) and heat pipes (HP) are not used widely. It have a simple mechanism, but nowadays common theory of heat transfer processes in such devices allowing describe for more complex physical processes in the areas of evaporation and condensation in the steam channel, a moving film of condensate is not developed [3,4].

Modern studies of heat transfer through a two-phase closed thermosyphon mainly represented by experimental study of heat and mass transfer processes [3-6], at the same time, the number of works involving mathematical modeling of these processes is insignificant $[7,8]$.

The purpose of work is mathematical modeling of heat transfer in two-phase thermosyphon rectangular cross-section with the package ANSYS FLUENT [6] and comparison between numerical modeling and experimental data.

\section{Physical model}

We consider the thermosyphon circuit diagram is shown in Figure 1.

${ }^{a}$ Corresponding author: krasnas@tpu.ru

This is an Open Access article distributed under the terms of the Creative Commons Attribution License 4.0, which permits unrestricted use, distribution, and reproduction in any medium, provided the original work is properly cited. 


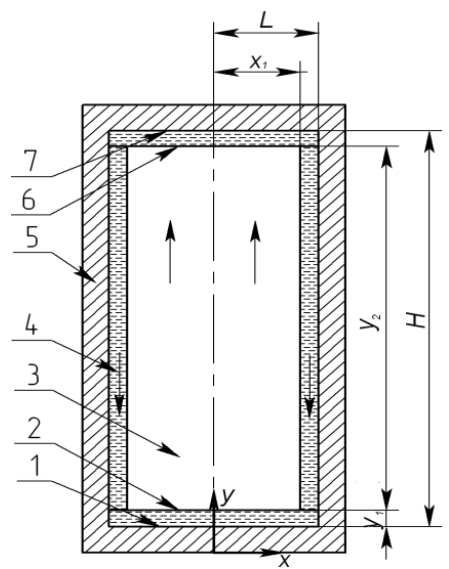

Figure 1. Chart of the closed thermosyphon. 1 - bottom cover; 2 - surface of evaporation; 3 - vapor flow; 4 film of liquid; 5 - wall of thermosyphon; 6 - surface of condensation; 7 - upper cover.

\section{Mathematical model and method of solution}

The equations of continuity, momentum and energy solved in ANSYS FLUENT [9] for vapor and liquid film in the study area are of the form:

$$
\begin{gathered}
\frac{\partial \rho}{\partial t}+\frac{\partial(\rho u)}{\partial x}+\frac{\partial(\rho v)}{\partial y}=0 \\
\frac{\partial \rho_{1} u_{1}}{\partial t}+\frac{\partial\left(\rho_{1} u_{1} u_{1}\right)}{\partial x}+\frac{\partial\left(\rho_{1} v_{1} u_{1}\right)}{\partial y}=\rho_{1} g_{x}-\frac{\partial P_{1}}{\partial x}+\frac{\partial}{\partial x}\left(\mu_{1} \frac{\partial u_{1}}{\partial x}\right)+\frac{\partial}{\partial y}\left(\mu_{1} \frac{\partial u_{1}}{\partial y}\right) \\
\frac{\partial \rho_{1} v_{1}}{\partial t}+\frac{\partial\left(\rho_{1} u_{1} v_{1}\right)}{\partial x}+\frac{\partial\left(\rho_{1} v_{1} v_{1}\right)}{\partial y}=\frac{\partial P_{1}}{\partial y}+\frac{\partial}{\partial x}\left(\mu_{1} \frac{\partial v_{1}}{\partial x}\right)+\frac{\partial}{\partial y}\left(\mu_{1} \frac{\partial v_{1}}{\partial y}\right) \\
\frac{\partial \rho_{2} u_{2}}{\partial t}+\frac{\partial\left(\rho_{2} u_{2} u_{2}\right)}{\partial x}+\frac{\partial\left(\rho_{2} v_{2} u_{2}\right)}{\partial y}=\rho_{2} g_{x}-\frac{\partial P_{2}}{\partial x}+\frac{\partial}{\partial x}\left(\mu_{2} \frac{\partial u_{2}}{\partial x}\right)+\frac{\partial}{\partial y}\left(\mu_{2} \frac{\partial u_{2}}{\partial y}\right) \\
\frac{\partial \rho_{2} v_{2}}{\partial t}+\frac{\partial\left(\rho_{2} u_{2} v_{2}\right)}{\partial x}+\frac{\partial\left(\rho_{2} v_{2} v_{2}\right)}{\partial y}=\frac{\partial P_{2}}{\partial y}+\frac{\partial}{\partial x}\left(\mu_{2} \frac{\partial v_{2}}{\partial x}\right)+\frac{\partial}{\partial y}\left(\mu_{2} \frac{\partial v_{2}}{\partial y}\right) \\
\rho_{1} C_{p 1}\left(\frac{\partial T_{1}}{\partial t}+u_{1} \frac{\partial T_{1}}{\partial x}+v_{1} \frac{\partial T_{1}}{\partial y}\right)=\lambda_{1}\left(\frac{\partial^{2} T_{1}}{\partial x^{2}}+\frac{\partial^{2} T_{1}}{\partial y^{2}}\right) \\
\rho_{2} C_{p 2}\left(\frac{\partial T_{2}}{\partial t}+u_{2} \frac{\partial T_{2}}{\partial x}+v_{2} \frac{\partial T_{2}}{\partial y}\right)=\lambda_{2}\left(\frac{\partial^{2} T_{2}}{\partial x^{2}}+\frac{\partial^{2} T_{2}}{\partial y^{2}}\right)
\end{gathered}
$$

where, $u_{1}, v_{1}$ - the velocity components in the projection on the axis $\mathrm{x}, \mathrm{y} ; \rho-$ density; $\mathrm{x}, \mathrm{y}-$ Cartesian coordinates; $\mathrm{t}$ - time; $\mathrm{Cp}$ - heat capacity; $\mathrm{g}$ - acceleration of gravity; $\lambda$ - coefficient of thermal conductivity; $\mu$-dynamic viscosity; indexes 1, 2 - property of the liquid and vapor.

The initial conditions for the system of equations (1-7):

$u(x, y)=0 ; T(x, y)=T_{0} ; P(x, y)=P_{0} ; t=0$. 
The boundary conditions for the equations (1-7) are determined:

$$
\begin{aligned}
& x=0,0<y<H, \lambda_{2} \frac{\partial T_{2}}{\partial x}=0 ; \frac{\partial u_{1}}{\partial x}=0 ; u_{1}=v_{1}=0 ; \\
& x=L, 0 \leq y \leq H, \lambda_{2} \frac{\partial T_{2}}{\partial x}=0 ; u_{2}=v_{2}=0 \\
& x=x_{1}, y_{1} \leq y \leq y_{1}+y_{2},\left\{\begin{array}{l}
T_{1}=T_{2} \\
\lambda_{1} \frac{\partial T_{1}}{\partial x}=\lambda_{2} \frac{\partial T_{2}}{\partial x}
\end{array} ;\left\{\begin{array}{l}
u_{1}=u_{2}=0 \\
v_{1}=v_{2}
\end{array}\right.\right. \\
& y=y_{1}, 0 \leq x \leq x_{1},\left\{\begin{array}{l}
T_{1}=T_{2} \\
\lambda_{2} \frac{\partial T_{2}}{\partial y}=\lambda_{1} \frac{\partial T_{1}}{\partial y}-Q_{e} w_{e}-C_{p} \rho\left(T_{1}-T_{0}\right), \quad \mathrm{v}_{1}=\frac{w_{e}}{\rho_{1}}, \mathrm{v}_{2}=0
\end{array}\right. \\
& y=y_{1}+y_{2}, 0 \leq x \leq x_{1},\left\{\begin{array}{l}
T_{1}=T_{2} \\
\lambda_{1} \frac{\partial T_{1}}{\partial y}=\lambda_{2} \frac{\partial T_{2}}{\partial y}+Q_{c} w_{c}+C_{p} \rho\left(T_{2}-T_{0}\right), \quad \mathrm{v}_{1}=\frac{w_{c}}{\rho_{1}}, \mathrm{v}_{2}=0,
\end{array}\right. \\
& y=0,0 \leq x \leq L, \lambda_{2} \frac{\partial T_{2}}{\partial y}=T_{h} ; u_{2}=v_{2}=0 \\
& y=H, 0 \leq x \leq L, \lambda_{2} \frac{\partial T_{2}}{\partial y}=q_{c}, u_{2}=v_{2}=0 \\
& y=y_{1}, x_{1} \leq x \leq L,\left\{\begin{array}{l}
T_{1}=T_{2} \\
\lambda_{2} \frac{\partial T_{2}}{\partial y}=\lambda_{1} \frac{\partial T_{1}}{\partial y}, \quad u_{2}=0 ; v_{2}=\frac{w_{c}}{\rho_{1}},
\end{array}\right. \\
& y=y_{1}+y_{2}, x_{1} \leq x \leq L,\left\{\begin{array}{l}
T_{1}=T_{2} \\
\lambda_{2} \frac{\partial T_{2}}{\partial y}=\lambda_{1} \frac{\partial T_{1}}{\partial y}, \quad u_{2}=0 ; v_{2}=\frac{w_{c}}{v_{1}} .
\end{array}\right.
\end{aligned}
$$

\section{Results and Discussion}

Numerical studies for the thermosyphon with longitudinal and transverse dimensions: $\mathrm{y}=350 \mathrm{~mm}, \mathrm{x}$ $=20 \mathrm{~mm}$ at the thermal loads on the bottom cover were performed: $1,5 \cdot 10^{5} \mathrm{~W} / \mathrm{m}^{2}, 3,8 \cdot 10^{5} \mathrm{~W} / \mathrm{m}^{2}$. The refrigerant used R600a. Selection of coolant due to the low operating pressure in comparison with other CFCs such as R134a.

From these temperature field, velocity vectors, streamlines shows that when $1,5 \cdot 10^{5} \mathrm{~W} / \mathrm{m}^{2}$ at the top of the thermosyphon is formed vortex (fig. 2a), caused by multidirectional movement hot vapor and chilled liquid film. On the figure $2 \mathrm{~b}$ shows that vortex changes insignificantly to the direction $y$ with increasing heat load $\mathrm{Q}=3,8 \cdot 10^{5} \mathrm{~W} / \mathrm{m}^{2}$. This is due to of increasing the speed difference vapor and liquid film. Also, in the evaporation zone in the border section of the steam passage and the condensate film formed a local circulating zone. It should be noted that eddy currents reduce the efficiency of heat transfer of closed two-phase thermosyphons. 

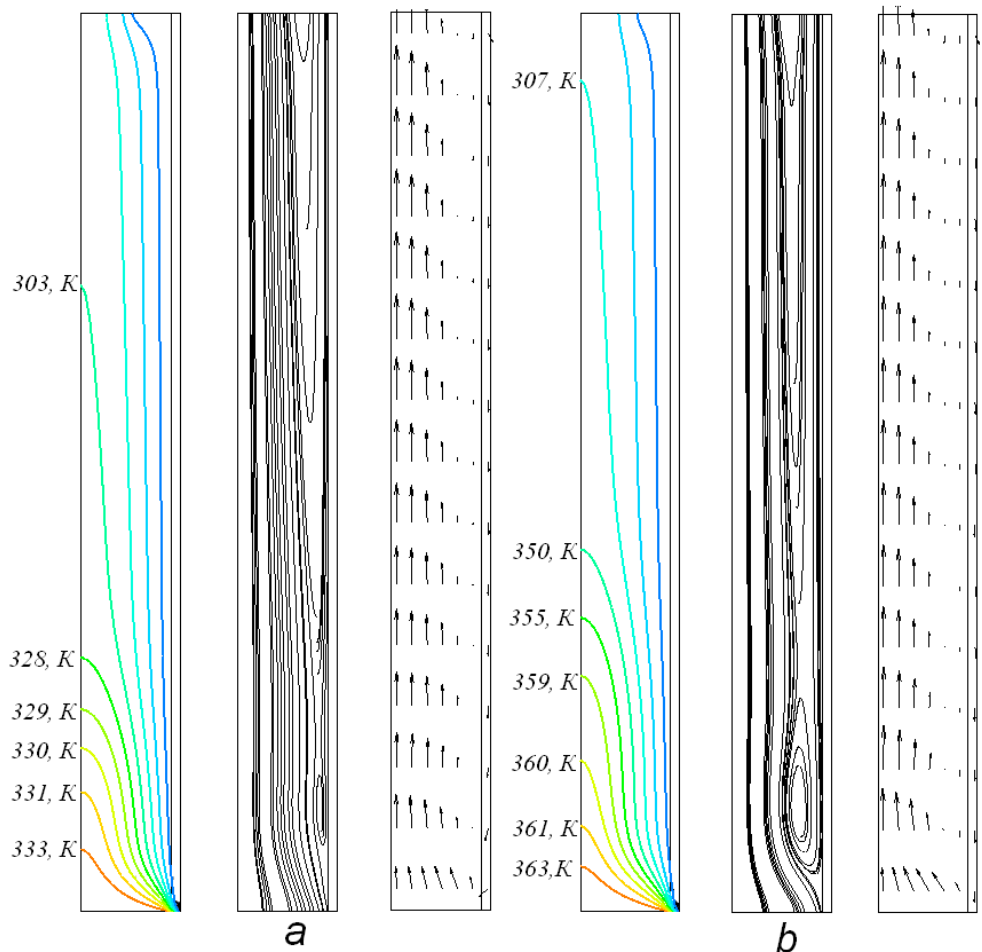

Figure 2. Temperature field, streamlines and velocity vectors in the study area. (a) $-1,5 \cdot 10^{5} \mathrm{~W} / \mathrm{m}^{2}$, (b) $-3,8 \cdot 10^{5}$ $\mathrm{W} / \mathrm{m}^{2}$.

The comparison (Fig.3) between numerical results and the experimental [6] confirms the accuracy of the simulations. The results of mathematical modeling are in good agreement with the experimental data.

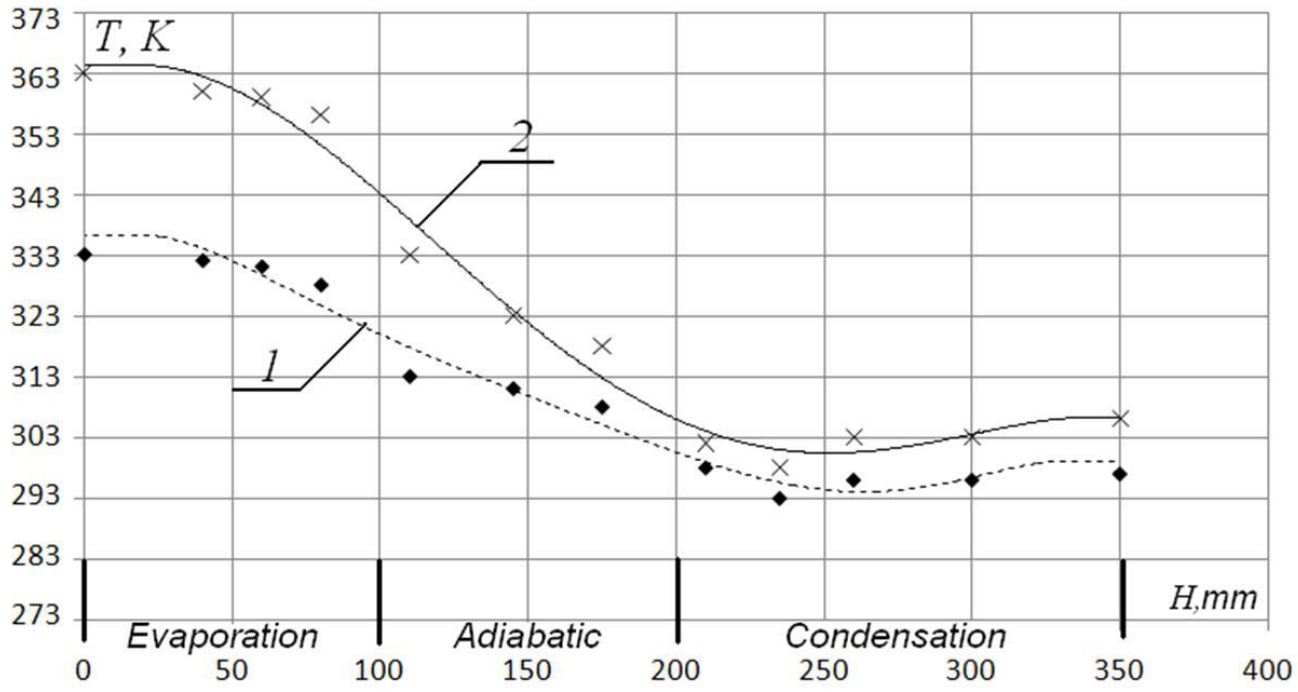

Figure 3.The temperature distribution in the direction y. The comparison between numerical results and the experimental.

Curve $1(\bullet)-1,5 \cdot 10^{5} \mathrm{~W} / \mathrm{m}^{2}$, curve $2(\searrow)-3,8 \cdot 10^{5} \mathrm{~W} / \mathrm{m}^{2}$. 


\section{Conclusion}

Mathematical modeling of heat transfer in two-phase thermosyphon rectangular cross-section with the package ANSYS FLUENT has shown the possibility of using this package in the analysis of energy transfer processes at work thermosyphons at high thermal loads. it was revealed The influence of heat flux supplied to the bottom cover on hydrodynamic characteristics of the study area. Comparison of the results of numerical investigation of the experimental data showed that their deviation was from 0,5 to $1,8 \%$.

The work was held within the research state assignment "Science” №13.1339.2014/K (Code of Federal Target Scientific and Technical Program 2.1410.2014).

\section{References}

1. M.K. Bezrodnyi, I.L. Pioro, T.S. Kostyuk, Transfer processes in the two-phase thermosyphon systems (Kiev, 2005) [in Russian]

2. M.K. Bezrodnyi, Two-phase thermosyphon industrial heating engineer (Kiev, 1991) [in Russian]

3. Hussam Jouhara, Anthony J. Robinson, Appl., 30 (2-3), 201 (2010)

4. V.Y. Kravetz, V.A. Chernobay, A.A. Nikitenko,East Europe Journal, 2/8 (50), 40 (2011)

5. A. Alizadehdakhel, M. Rahimi, Heat Mass Transfer, 312 (2010)

6. Brusly Solomon A., Roshan R., Int. J. Heat Mass Transfer, 82, 521 (2015)

7. G.V.Kuznetsov, A.E. Sitnikov, High Temperature, 40 (6), 898 (2002)

8. G.V. Kuznetsov, M.A. Al-Ani, M.A. Sheremet, Journal of Engineering Thermophysics, 20 (2), 201 (2011)

9. Ansys Help. FLUENT Theory Guide 